\title{
Simulating a Maxwellian plasma using an electron beam ion trap
}

\author{
D. W. Savin a) \\ Columbia Astrophysics Laboratory and Department of Physics, Columbia University, New York, \\ New York 10027 \\ P. Beiersdorfer \\ Department of Physics and Space Technology, Lawrence Livermore National Laboratory, Livermore, \\ California 94550 \\ S. M. Kahn \\ Columbia Astrophysics Laboratory and Department of Physics, Columbia University, New York, \\ New York 10027 \\ B. R. Beck and G. V. Brown \\ Department of Physics and Space Technology, Lawrence Livermore National Laboratory, Livermore, \\ California 94550 \\ M. F. Gu \\ Columbia Astrophysics Laboratory and Department of Physics, Columbia University, New York, \\ New York 10027 \\ D. A. Liedahl and J. H. Scofield \\ Department of Physics and Space Technology, Lawrence Livermore National Laboratory, Livermore, \\ California 94550
}

(Received 23 February 2000; accepted for publication 17 May 2000)

We describe a technique for producing a Maxwell-Boltzmann electron energy distribution using an electron beam ion trap (EBIT). The technique was implemented on the Lawrence Livermore EBIT to simulate Maxwellian plasmas. We discuss technical and experimental issues related to these simulations. To verify the fidelity of the quasi-Maxwellian, we have measured line emission due to dielectronic recombination (DR) and electron impact excitation (EIE) of heliumlike neon, magnesium, and argon for a range of simulated electron temperatures. The ratio of DR to EIE lines in heliumlike ions is a well understood electron temperature diagnostic. The spectroscopically inferred quasi-Maxwellian temperatures are in excellent agreement with the simulated temperatures.

(C) 2000 American Institute of Physics. [S0034-6748(00)00609-2]

\section{INTRODUCTION}

Many laboratory and astrophysical plasmas involve a Maxwell-Boltzmann distribution of electrons colliding with an ensemble of ions. Accurately modeling and interpreting the photon emission and ionization structure of these Maxwellian plasmas requires that the electron-ion collisional rate coefficients be known for tens of thousands of excitation, ionization, and recombination processes. Theoretical calculations provide the majority of the cross sections from which the necessary rate coefficients are derived. But approximations often need to be made to make the calculations tractable. Experiments can provide benchmark cross section measurements to test these various approximations. However, carrying out such measurements over the energy range required to calculate accurate rate coefficients is often prohibitively time consuming. The analog solution to this problem is to design an experiment with a Maxwell-Boltzmann electron distribution. This automatically integrates the collision cross sections with the desired electron distribution, and the observed properties of ions in such experiments are directly dependent on the relevant rate coefficients.

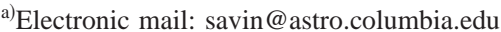

Tokamaks and $\theta$ pinches are two of the most common laboratory Maxwellian plasmas used for measuring electron-ion collisional rate coefficients. A fair number of excitation, ionization, and recombination rate coefficients have been determined using these devices. ${ }^{1}$ The accuracy of these measurements, however, is often limited by complications such as density effects, radiative transfer, ion abundance gradients, the electron temperature and density structures of the plasmas, and line-of-sight averaging of the observed photon emission over regions of multiple electron temperatures.

Here we present a new laboratory technique for studying ions interacting with a Maxwell-Boltzmann electron distribution. Using the Lawrence Livermore electron beam ion trap $(\text { EBIT })^{2,3}$ we have produced a quasi-Maxwellian plasma by sweeping the energy of the nearly monoenergetic beam so that the time spent at any one energy is proportional to the Maxwell-Boltzmann electron distribution probability at that energy.

EBIT offers a number of advantages over standard plasma sources. The resulting EBIT plasma is essentially driven by a Maxwellian electron distribution at a single temperature $T_{e}$. Because the operating parameters of EBIT can 
be easily changed, a wide range in $T_{e}$ can be simulated. Density effects are generally unimportant. The plasma is optically thin. And the electron temperature structure is essentially uniform along the line of sight. Another advantage is the capacity to create ions of a given charge state and then study them in a Maxwellian plasma under nonequilibrium conditions.

To verify the accuracy of our simulated MaxwellBoltzmann distribution, we have carried out measurements of line emission due to dielectric recombination (DR) and electron impact excitation (EIE) of heliumlike neon, magnesium, and argon. Heliumlike ions are commonly used to measure the electron temperature of a plasma by taking the ratio of DR produced lines to EIE lines. ${ }^{4}$

In Sec. II we present those aspects of EBIT relevant to the Maxwellian simulation. In Sec. III we describe the specific technique we use to simulate a Maxwell-Boltzmann electron distribution, implementation of the technique, and how to interpret the resulting line emission. In Sec. IV we discuss the experimental uncertainties associated with our measurements. Discussed in Sec. V are the various theoretical calculations used to verify the accuracy of the quasiMaxwellian simulations. In Sec. VI we present our results and compare with theoretical predictions.

\section{APPARATUS}

The Lawrence Livermore EBIT uses a magnetically confined, vertically directed beam of electrons to produce and trap highly charged ions. ${ }^{2,3}$ The electron beam is formed using a Pierce gun. ${ }^{5}$ The beam density can be varied between $\sim 10^{11}$ and $10^{13} \mathrm{~cm}^{-3}$. The beam is approximately Gaussian in shape with a radius of $\sim 30-35 \mu \mathrm{m}$. ${ }^{3,6,7}$ The beam energy can be varied between $\sim 0.2$ and $20.0 \mathrm{keV}$. Below $\sim 0.2$ $\mathrm{keV}$, the electron beam is poorly behaved and does not trap ions well. Above $\sim 15 \mathrm{keV}$, voltage breakdowns begin to occur inside EBIT unless the machine is properly conditioned. The energy spread of the electron beam for typical operating parameters is $\sim 35-50 \mathrm{eV} ., 8,9$ For sufficiently high energies, the electron beam is nearly monoenergetic. The beam energy $E$ and electron gun anode (extraction) potential $V_{a}$ are controlled using separate Trek 20/20 high voltage power supplies. In the trapping region, $E$ is determined by the applied potentials plus space charge corrections.

The electron beam space charge $V_{e}$ in EBIT is given in volts by $V_{e} \sim 5 I_{e} / E^{1 / 2}$ where $I_{e}$ is the beam current in milliamperes at energy $E$, which is given in kilovolts. ${ }^{10}$ For typical operating conditions $V_{e}$ is $\sim-200 \mathrm{~V}$. The space charge of the trapped ions $V_{i}$ is $\sim-V_{e} / 2$. The resulting total space charge is $V_{e}+V_{i} \sim V_{e} / 2$, typically $\sim-100 \mathrm{~V}$. We apply an additional positive potential to cancel out most of this space charge.

Current limitations of the power supplies driving EBIT and capacitances in EBIT limit the slew rate of $E$ to $\$ 30$ $\mathrm{V} \mu \mathrm{s}^{-1} . V_{a}$ must be driven at slew rates $\leqslant 30 \mathrm{~V} \mu \mathrm{s}^{-1}$ to minimize ringing of the applied voltage.

For the present experiments, we have used vacuum flat crystal spectrometers (FCSs) ${ }^{11-13}$ and a windowless lithiumdrifted silicon $[\mathrm{Si}(\mathrm{Li})]$ solid-state detector to measure the x-ray spectra. The dispersion plane of each FCS is perpendicular to the magnetic field that confines the electrons. The $\mathrm{Si}(\mathrm{Li})$ detector angle of observation is also perpendicular to the magnetic field. The spectrometers view the central $1 \mathrm{~cm}$ length of the trap. There is a window between EBIT and each FCS. Various windows $(1 / 2 \mu \mathrm{m}$ Lexan, $1 \mu \mathrm{m}$ Lexan, 1 $\mu \mathrm{m}$ Mylar, and $1 / 2 \mu \mathrm{m}$ polyimide) were used. $\mathrm{X}$ rays are detected using flowing gas proportional counters $(90 \% \mathrm{Ar}$ and $10 \% \mathrm{CH}_{4}$ at $\left.\sim 1 \mathrm{~atm}\right),{ }^{14}$ with $4 \mu \mathrm{m}$ polypropylene windows coated with 200-400 $\AA$ of aluminum. The depth of the absorbing gas volume in each detector is $\sim 9 \pm 1 \mathrm{~mm}$. A series of vertical apertures collimates the emission observed by each FCS for a maximum divergence of $\leqslant 18 \mathrm{mrad}$ in the vertical dimension.

\section{EXPERIMENTAL TECHNIQUE}

\section{A. Simulating a Maxwell-Boltzmann energy distribution}

It is possible to simulate a Maxwell-Boltzmann electron energy distribution by sweeping an electron beam in energy and current as a function of time. The Maxwell-Boltzmann probability of finding an electron in the energy range $E$ to $E+d E$ is given by

$$
P\left(E, T_{e}\right) d E=\frac{2 E^{1 / 2}}{\pi^{1 / 2}\left(k_{B} T_{e}\right)^{3 / 2}} \exp \left(\frac{-E}{k_{B} T_{e}}\right) d E,
$$

where $k_{B}$ is the Boltzmann constant. Using a monoenergetic electron beam, one may simulate a Maxwell-Boltzmann distribution by maintaining a constant electron density and sweeping the instantaneous energy $E$ in time so that the fraction of time the beam energy is in the range $E^{\prime}$ to $E^{\prime}$ $+d E^{\prime}$ equals $P\left(E^{\prime}, T_{e}\right) d E^{\prime}$. This condition can be expressed as

$$
\frac{d \tau}{\tau_{0}}=P\left(E^{\prime}, T_{e}\right) d E^{\prime},
$$

where $\tau_{0}$ is the length of the sweep pattern. Solving Eq. (2) for $\tau$ as a function of $E$ (see the Appendix) we find

$$
\tau(E)=\tau_{0}\left[\operatorname{erf}(x)-\frac{2 x e^{-x^{2}}}{\sqrt{\pi}}\right],
$$

where $\operatorname{erf}(x)$ is the error function, $x=\left(E / k_{b} T_{e}\right)^{1 / 2}$, and the quantity in the square brackets ranges between 0 and 1 . The electron energy sweep pattern $E(\tau)$ may be calculated numerically using Eq. (3).

As discussed in Sec. III B, the electron density is a function of the $E$ and $V_{a}$. Normally we attempt to maintain a constant electron density. But at times it may be desirable to maintain a constant $V_{a}$ and allow the electron density $n_{e}$ to vary. In such cases, the sweep pattern must be modified. For $n_{e}=n_{0} f(E)$, where $n_{0}$ is the density at energy $E_{0}$ and $f\left(E_{0}\right)=1.0$, Eq. (3) becomes

$$
\tau(E)=\frac{\tau_{0}}{f(E)}\left[\operatorname{erf}(x)-\frac{2 x e^{-x^{2}}}{\sqrt{\pi}}\right] .
$$




\section{B. Maintaining a constant-density electron beam}

The electron beam density may be kept constant by sweeping $V_{a}$ synchronously with $E(\tau)$. The current output from a Pierce gun is given in amperes by ${ }^{5}$

$$
I_{e}=p V_{a}^{3 / 2},
$$

where $p$ is the perveance in units of $\mathrm{A} \mathrm{V}^{-3 / 2}$ and $V_{a}$ is in volts. For a beam of constant shape and size, we have

$$
n_{e} \propto \frac{V_{a}^{3 / 2}}{E^{1 / 2}} .
$$

To keep $n_{e}$ constant as a function of $E(\tau), V_{a}$ is swept so that

$$
V_{a}(\tau)=\left(V_{a}\right)_{r}\left[\frac{E(\tau)}{E_{r}}\right]^{1 / 3},
$$

where $\left(V_{a}\right)_{r}$ is the anode voltage at an arbitrary reference energy $E_{r}$. Normally we choose $\left(V_{a}\right)_{r}=\left(V_{a}\right)_{\min }$ and $E_{r}$ $=E_{\min }$, where $\left(V_{a}\right)_{\min }$ is the lowest anode voltage used for the $V_{a}(\tau)$ timing pattern and $E_{\min }$ is the lowest beam energy used for the $E(\tau)$ timing pattern.

\section{Implementation using an electron beam ion trap}

Using the EBIT, we produce a quasi-Maxwellian plasma by maintaining a nearly constant $n_{e}$ while sweeping $E$ between $E_{\min } \sim 0.2 \mathrm{keV}$ and $E_{\max } \sim 5-6 k_{B} T_{e}$. The high energy cutoff is chosen so that $E_{\max } \lesssim 15 \mathrm{keV}, d E / d t \lesssim 30 \mathrm{~V} \mu \mathrm{s}^{-1}$, and typically less than $2 \%$ of the Maxwellian distribution is lost. Effects of $E_{\min }$ and $E_{\max }$ on the equilibrium time and line emission of the quasi-Maxwellian plasma are discussed in Sec. IV C.

Due to the $E_{\min }$ and $E_{\max }$ limitation, we do not sweep over the entire period $\tau_{0}$. The actual sweep period is given by $t_{0}=\tau\left(E_{\max }\right)-\tau\left(E_{\min }\right)$. The specific time versus $E$ in the applied sweep pattern $E(t)$ is given by $t(E)=\tau(E)$ $-\tau\left(E_{\min }\right)$.

To avoid problems of trying to sweep faster than the slew rate of the EBIT electrical system, we sweep from $E_{\text {min }}$ to $E_{\max }$ and then back down to $E_{\min }$ using the same pattern as the upsweep but mirrored around $t=t_{0}$. For the $T_{e}$ range we are interested in simulating, the maximum slew rate for $E$ limits $t_{0}$ to values $\gtrsim 5 \mathrm{~ms}$.

We attempt to maintain a constant $n_{e}$, partially to keep the EBIT trapping conditions unchanged during a sweep. The electron beam is tuned using an $E_{\min }$ as low as possible, so that we cut out as little of the Maxwellian distribution as possible. $\left(V_{a}\right)_{\min }$ is tuned as high as possible in order to achieve a high beam density. This procedure results in better trapping conditions and an increased signal rate. Equation (7) is used to determine $V_{a}(t)$.

$E(t)$ and $V_{a}(t)$ are generated using a LeCroy 9109, programmable, two channel arbitrary function generator (AFG). The clock period of the AFG is $10 \mathrm{~ns}$. Each AFG channel is limited to a maximum of 256 voltage steps. A program has been written to digitize the wave forms given by Eqs. (3) and (7). The energy range $E_{\min }$ to $E_{\max }$ is divided into 256 equal steps. Taking into account the constraints on $E_{\max }$ and

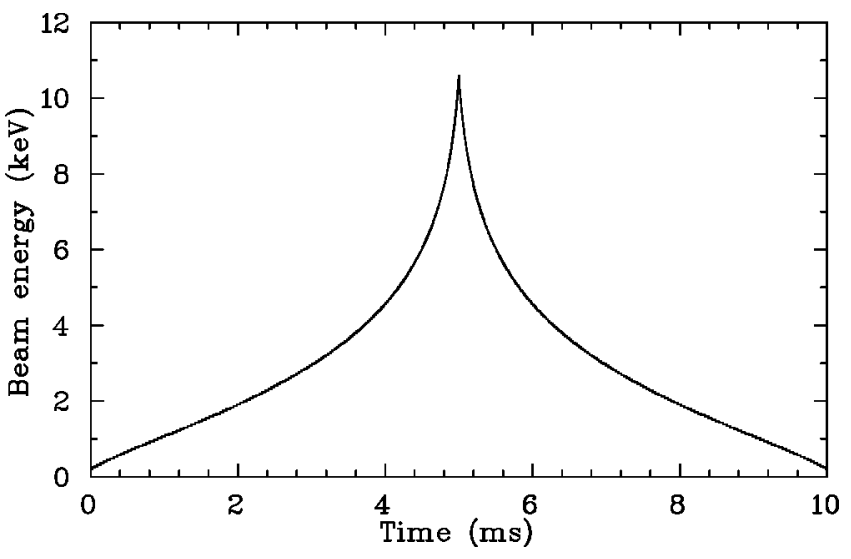

FIG. 1. Digitized timing pattern of the electron beam energy used for simulating a Maxwellian plasma at $T_{e}=2.0 \mathrm{keV}$. Representative operating conditions of $E_{\min }=0.2 \mathrm{keV}, E_{\max }=10.6 \mathrm{keV}$, and $t_{0}=5 \mathrm{~ms}$ have been used.

$d E / d t$, we choose $E_{\max }$ so that $\left(E_{\max }-E_{\min }\right) / 256$ is smaller than the $\sim 35-50 \mathrm{eV}$ energy spread of the electron beam. The capacitance of EBIT and the beam energy spread, caused by ripple in the power supply and the gradient in the space charge of the electron beam, reduce somewhat the effects of the discrete energy steps in $E(t)$. Thus, the time varying $E$ of the electrons in EBIT is actually a smoothed approximation of the applied $E(t)$. The voltage range $V_{a}\left(E_{\min }\right)$ to $V_{a}\left(E_{\max }\right)$ is also divided into 256 equal steps. Design limitations of the EBIT electron gun require that we keep $V_{a}<5.0 \mathrm{keV}$. Hence, the $V_{a}(t)$ voltage steps are $\ 20$ V.

A representative digitized $E(t)$ for simulating a Maxwellian at a temperature of $2.0 \mathrm{keV}$ is shown in Fig. 1. The corresponding digitized $V_{a}(t)$ for maintaining a constant $n_{e}$ is shown in Fig 2. Figures 1 and 2 do not include the constant $\sim+100 \mathrm{~V}$ applied to cancel space charge effects.

\section{Line ratio measurements}

Here in Sec. III D we develop expressions for the measured and predicted line intensities from EBIT for quasi-

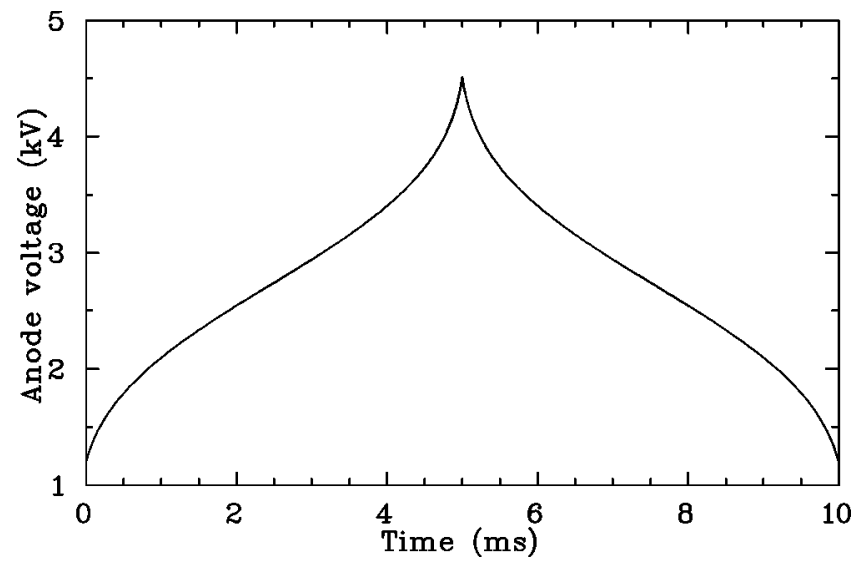

FIG. 2. Digitized timing pattern of the electron gun anode voltage used to generate a constant density electron beam in EBIT while simulating a Maxwellian plasma at a temperature of $2.0 \mathrm{keV}$. Representative operating conditions of $E_{\min }=0.2 \mathrm{keV}, E_{\max }=10.6 \mathrm{keV},\left(V_{a}\right)_{\min }=1.2 \mathrm{kV}$, and $t_{0}=5 \mathrm{~ms}$ have been used. 
Maxwellian simulations. We begin by assuming a monoenergetic beam of unidirectional electrons and an ensemble of trapped, ground state ions of charge $q$. The resulting line emission from EBIT can be expressed in units of photons $\mathrm{s}^{-1}$ as

$$
I=\sigma(E) v_{e}(E) \int n_{e}(\mathbf{r}) n_{q}(\mathbf{r}) d^{3} \mathbf{r},
$$

where $E$ is the collision energy (essentially the electron energy); $\sigma$ is the cross section for starting with a ground state ion and collisionally producing the line of interest (including branching ratios and cascade contributions); $v_{e}$ is the electron velocity; $n_{e}(\mathbf{r})$ is the electron density at $\mathbf{r} ; n_{q}(\mathbf{r})$ is the ion density at $\mathbf{r}$; and $\int d^{3} \mathbf{r}$ is over the volume of the trap. Excited ions are assumed to decay radiatively on a timescale significantly shorter than either the trapping time of the ions or the timescale over which the charge balance in the trap changes. The energy dependences of $\sigma$ and $v_{e}$ and spatial dependences of $n_{e}$ and $n_{q}$ are hereafter implicitly assumed.

A unidirectional beam of electrons colliding with atomic or ionic targets may produce anisotropically emitted, polarized radiation. ${ }^{15-17}$ The intensity from EBIT of an electric dipole line measured using a FCS can be written ${ }^{8,18}$

$$
I^{\mathrm{FCS}}=T D \psi G\left(\frac{I}{4 \pi}\right),
$$

where $T$ accounts for the x-ray transmittances of all windows, $D$ is the x-ray detection efficiency, $\psi$ is the vertical angle collected by the FCS, $I$ is given by Eq. (8), and

$$
G=\frac{3\left[\left(R_{\sigma}+R_{\pi}\right)+\left(R_{\sigma}-R_{\pi}\right) \mathcal{P}\right]}{2(3-\mathcal{P})} .
$$

Here $R_{\sigma}$ and $R_{\pi}$ are, respectively, the integrated crystal reflectivities for $\mathrm{x}$ rays polarized perpendicular to and parallel to the dispersion plane of the FCS. $\mathcal{P}$ is the polarization of the line. The polarization may be energy dependent and is given by ${ }^{15-17}$

$$
\mathcal{P}=\frac{I_{\sigma}-I_{\pi}}{I_{\sigma}+I_{\pi}},
$$

where $I_{\sigma}$ and $I_{\pi}$ are measured at an observation angle perpendicular to the electron beam and are, respectively, the intensity of line emission polarized parallel to and perpendicular to the beam direction.

For the windowless $\mathrm{Si}(\mathrm{Li})$ detector the measured intensity is given by

$$
I^{\mathrm{Si}}=G^{\mathrm{Si}} D d \Omega\left(\frac{I}{4 \pi}\right),
$$

where $d \Omega$ is the solid angle collected by the detector and $G^{\mathrm{Si}}$ is given by

$$
G^{\mathrm{Si}}=\frac{3}{(3-\mathcal{P})} .
$$

For quasi-Maxwellian simulations as described above, in a period $t_{0}$ the total line emission from EBIT for a given charge state can be expressed in units of photons $\mathrm{s}^{-1}$ as

$$
I=\frac{1}{t_{0}} \int_{0}^{t_{0}} \sigma v_{e} d t \int n_{e} n_{q} d^{3} \mathbf{r},
$$

where, in addition to our previous assumptions, we also assume $\int n_{e} n_{q} d^{3} \mathbf{r}$ is constant versus $t$ (i.e., versus $E$ ) and that the beam energy is swept rapidly enough so that the ionization balance of the trapped ions is nearly constant over one period of the energy sweep. Using our definition of $t$ and Eq. (2), we can rewrite Eq. (14) as

$$
I=\int_{E_{\min }}^{E_{\max }} \sigma v_{e} P\left(E, T_{e}\right) d E \int n_{e} n_{q} d^{3} \mathbf{r} .
$$

If the formation of the line of interest is insignificant outside the energy range $E_{\min }-E_{\max }$, then Eq. (15) can be accurately approximated as

$$
I\left(T_{e}\right)=C\left(T_{e}\right) \int n_{e} n_{q} d^{3} \mathbf{r},
$$

where $C\left(T_{e}\right)$ is the rate coefficient for the line of interest and is given by

$$
C\left(T_{e}\right)=\int \sigma v_{e} P\left(E, T_{e}\right) d E .
$$

Similar to Eq. (9), for quasi-Maxwellian simulations the intensity of a line measured using a FCS can be written

$$
I^{\mathrm{FCS}}=T D \psi G^{\prime}\left(\frac{I}{4 \pi}\right),
$$

where $I$ is given by Eq. (15) or (16) and

$$
G^{\prime}=\frac{\int_{E_{\min }}^{E_{\max }} \sigma v_{e} G P\left(E, T_{e}\right) d E}{\int_{E_{\min }}^{E_{\max }} \sigma v_{e} P\left(E, T_{e}\right) d E} .
$$

If the observed feature is composed of more than one line, then $G^{\prime}$ becomes

$$
G^{\prime}=\frac{\sum_{i} \int_{E_{\min }}^{E_{\max }} \sigma_{i} v_{e} G_{i} P\left(E, T_{e}\right) d E}{\sum_{i} \int_{E_{\min }}^{E_{\max }} \sigma_{i} v_{e} P\left(E, T_{e}\right) d E},
$$

where the sum over $i$ accounts for all unresolved lines in the feature. Using a single FCS, line ratios determined from two simultaneously observed lines are compared to predicted line ratios using Eq. (18). For situations where Eq. (16) is valid, then the ratio of two lines, 1 and 2, produced by electron collisions with the same initial charge state is given by

$$
\frac{I_{1}}{I_{2}}=\frac{C_{1}}{C_{2}}=\frac{T_{2} D_{2} G_{2}^{\prime} I_{1}^{\mathrm{FCS}}}{T_{1} D_{1} G_{1}^{\prime} I_{2}^{\mathrm{FCS}}} .
$$

Equations (9), (12), and (18) have been derived for a unidirectional beam of electrons. The electrons in EBIT, however, are not truly unidirectional. They possess a velocity component $\mathbf{v}_{\perp}$ which is perpendicular to the confining magnetic field. As a result, they spiral around the magnetic field with an average pitch angle $\theta$. This spiraling can alter the pattern of the emitted radiation. As has been shown elsewhere, ${ }^{19,20}$ for electric dipole transitions the effects of this spiraling can be accounted for by replacing the polarization factor in Eqs. (10) and (13) by 
TABLE I. Fit parameters for RR into the $1 s$ subshell $\mathrm{of} \mathrm{Ar}^{17+}$ and $\mathrm{Ar}^{18+}$. Parameters are given for the theoretical total cross section, differential cross section for photon emission at $90^{\circ}$, and polarization of photons emitted at $90^{\circ}$.

\begin{tabular}{cccccccc}
\hline \hline & \multicolumn{9}{c}{$\mathrm{Ar}^{17+}$} & & \multicolumn{3}{c}{$\mathrm{Ar}^{18+}$} \\
\cline { 2 - 3 } Parameter & $\begin{array}{c}\sigma \\
\left(10^{-24} \mathrm{~cm}^{2}\right)\end{array}$ & $\begin{array}{c}d \sigma / d \Omega \\
\left(10^{-24} \mathrm{~cm}^{2} \mathrm{sr}^{-1}\right)\end{array}$ & $\mathcal{P}$ & & $\begin{array}{c}\sigma \\
\left(10^{-24} \mathrm{~cm}^{2}\right)\end{array}$ & $\begin{array}{c}d \sigma / d \Omega \\
\left(10^{-24} \mathrm{~cm}^{2} \mathrm{sr}^{-1}\right)\end{array}$ & $\mathcal{P}$ \\
\hline$a$ & 640 & 75.9 & 1 & 307 & 36.4 & 1 \\
$b$ & 1.14 & 1.15 & $-1.89 e-5$ & 1.14 & 1.15 & $-1.05 e-5$ \\
$c$ & $6.36 e-2$ & $6.86 e-2$ & $-1.14 e-4$ & & $6.30 e-2$ & $6.78 e-2$ & $-1.12 e-4$ \\
$d$ & $1.13 e-2$ & $1.23 e-2$ & & $1.12 e-2$ & $1.22 e-2$ & \\
\hline \hline
\end{tabular}

$$
\mathcal{P}=\mathcal{P}_{0} \frac{2-3 \epsilon}{2-\epsilon \mathcal{P}_{0}} .
$$

Here $\mathcal{P}_{0}$ is the polarization for a nonspiraling electron beam and $\epsilon=\sin ^{2} \theta=E_{\perp} / E$, where $E_{\perp}$ is the energy component due to $\mathbf{v}_{\perp}$. The value of $E_{\perp}$ depends on beam tuning parameters of EBIT but can be estimated for optimal conditions. A derivation using adiabatic invariants gives ${ }^{21,22}$

$$
E_{\perp}=E_{\perp c}\left(\frac{r_{c}}{r_{b}}\right)^{2},
$$

where $E_{\perp c}$ is the transverse energy of the electrons at the cathode, $r_{c}$ the radius of the beam at the cathode, and $r_{b}$ the radius in the trapping region. $E_{\perp c}$ is roughly given by $k_{B} T_{c}$, where $T_{c}$ is the cathode temperature. For $r_{c}=1.5 \mathrm{~mm}, r_{b}$ $\sim 30-35 \mu \mathrm{m}$, and $k_{B} T_{c} \sim 0.1 \mathrm{eV},{ }^{2,3,23}$ we find $E_{\perp} \sim 200 \mathrm{eV}$.

\section{UNCERTAINTIES}

\section{A. Electron-ion overlap}

We have investigated the energy dependence of the electron-ion overlap $\int n_{e} n_{q} d^{3} \mathbf{r}$ by observing radiative recombination (RR) into the $n=1$ shells of bare and hydrogenic argon. Neutral argon is injected into EBIT and ionized using a beam energy of $12.0 \mathrm{keV}$. We apply a linear voltage ramp from 12.0 to $0.5 \mathrm{keV}$ and back up to $12.0 \mathrm{keV}$. The period of the sweep is $1 \mathrm{~ms}$ down and $1 \mathrm{~ms}$ up. The down-up pattern is repeated for a total of $8 \mathrm{~ms}$, followed by $7 \mathrm{~ms}$ of constant beam energy at $12.0 \mathrm{keV}$. The entire $15 \mathrm{~ms}$ pattern is repeated for $\sim 1 \mathrm{~s}$ and then the ions are dumped. The electron density is kept constant using Eq. (7). RR photons are collected using a $\mathrm{Si}(\mathrm{Li})$ detector which is predicted to have a nearly constant efficiency over the energy range of the observed photons. The photon energy, the electron beam energy, and the time of each event are recorded using an "event-mode" data acquisition scheme. ${ }^{24}$

For electron energies $\$ 3.0 \mathrm{keV}, \mathrm{RR}$ emits photons essentially in a dipole pattern, and the electron-ion overlap can be written

$$
\int n_{e} n_{q} d^{3} \mathbf{r}=\frac{4 \pi I^{\mathrm{Si}}}{\sigma v_{e} G^{\mathrm{Si}} D d \Omega} .
$$

At higher energies, this approximation begins to break down, and it is more accurate to use the differential RR cross section for photon emission at $90^{\circ}$. The electron-ion overlap can then be written

$$
\int n_{e} n_{q} d^{3} \mathbf{r}=\frac{I^{\mathrm{Si}}}{\frac{d \sigma}{d \Omega} v_{e} D d \Omega}
$$

Photons due to $\mathrm{RR}$ onto $\mathrm{Ar}^{17+}$ and $\mathrm{Ar}^{18+}$ are not well resolved by the $\mathrm{Si}(\mathrm{Li})$ detector. We use the sum of the counts in the two RR features to determine $I^{\mathrm{Si}}$ vs $E$.

Theoretical total and differential RR cross sections have been calculated using a Hartree-Slater model ${ }^{25,26}$ which is in good agreement with photoionization experiments. ${ }^{27} \mathrm{We}$ have fit the theoretical total and differential cross sections to the formula

$$
\sigma, \frac{d \sigma}{d \Omega}=a \exp \left(-b \gamma-c \gamma^{2}-d \gamma^{3}\right)
$$

where $\gamma=\ln (E)$ and $E$ is in $\mathrm{keV}$. We have fit the polarizations using

$$
\mathcal{P}=a+b \gamma+c \gamma^{2}
$$

where the coefficients $a, b$, and $c$ in Eq. (27) are not the same as those in Eq. (26). The fit parameters are given in Table I. All fits are valid for $0.1 \leqslant E \leqslant 15.5 \mathrm{keV}$. The cross section fits are good to better than $1 \%$ and the polarization fits to better than $0.05 \%$. For energies between 0.1 and $15.5 \mathrm{keV}$, the relative energy dependence of the differential cross sections for $\mathrm{Ar}^{17+}$ and $\mathrm{Ar}^{18+}$ differ by $\lesssim 2.5 \%$. We use the calculated total and differential cross section for RR onto $\mathrm{Ar}^{17+}$ in Eqs. (24) and (25).

Figure 3 shows the measured energy dependence of the

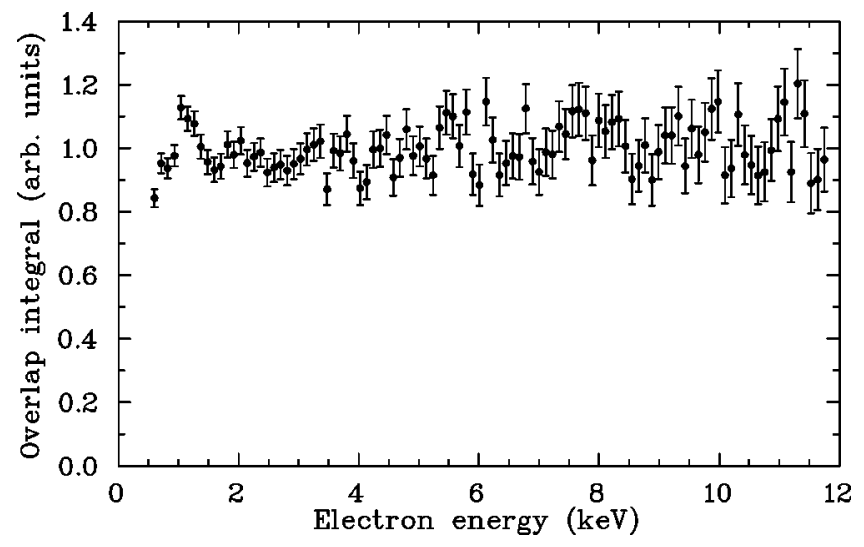

FIG. 3. Measured electron-ion overlap integral vs beam energy. One sigma statistical error bars are shown. Data were collected for $\sim 20 \mathrm{~h}$ and included $\mathrm{Ar}^{17+}$ and $\mathrm{Ar}^{18+}$ ions. 
electron-ion overlap integral [Eq. (25)] in arbitrary units. Electron spiraling has been accounted for for $E \leqslant 3.0 \mathrm{keV}$ assuming $E_{\perp} \sim 200 \mathrm{eV}$. Above $3.0 \mathrm{keV}$, the effects of electron spiraling are negligible. For $E$ between 1.5 and 11.7 $\mathrm{keV}$, the overlap is constant to within $\sim 8 \%$, at a $1 \sigma$ confidence level. Between 0.6 and $1.5 \mathrm{keV}$ there seems to be nonrandom behavior, but the overlap can be considered constant to within $\sim \pm 11 \%$. We are unable to determine the overlap integral for $E \leqq 0.6 \mathrm{keV}$ because the RR features begin to blend with $n \rightarrow 1$ lines formed by charge transfer of $\mathrm{Ar}^{17+}$ and $\mathrm{Ar}^{18+}$ with background gas. The electron beam is poorly behaved for $E \leqq 0.6 \mathrm{keV}$; but since the DR and EIE lines we are interested in here are produced at $E \gtrsim 0.68 \mathrm{keV}$, this behavior does not affect the measured line intensities.

Recent electron beam radius $r_{e}$ measurements have been carried out for $E \geqslant 17 \mathrm{keV}$ (Ref. 7) using the high-energy Livermore Super-EBIT. ${ }^{28}$ These measurements show that for constant $E, r_{e}$ (and hence $n_{e}$ ) varies as a function of beam current (i.e., versus $V_{a}$ ), and for constant $V_{a}, r_{e}$ is independent of $E$. It is not known how these results scale down to our beam energies. It is likely that $r_{e}$ and $n_{e}$ do in fact change as we vary $V_{a}$ in an attempt to maintain a constant $n_{e}$ vs $E$. However, for the Maxwellian simulations the important quantity is actually the electron-ion overlap factor, which our RR results indicate is constant to within $\pm 11 \%$ for 0.6 $\lesssim E \lesssim 11.7 \mathrm{keV}$. This constancy is most likely due to the ion density being nearly uniform over the spatial regions sampled by the electron beam.

The RR data were collected using a ramp of $1 \mathrm{~ms}$. Quasi-Maxwellian data have been collected using a ramp of $5 \mathrm{~ms}$. Heating of the ions in $5 \mathrm{~ms}$ is predicted to be insignificant ${ }^{29}$ and we expect $n_{q}$ to remain essentially constant during the Maxwellian voltage ramps. We therefore infer that the measured energy dependence of the overlap integral is appropriate also for our quasi-Maxwellian simulations.

\section{B. Line intensities}

Measurements have been carried out using magnesium, neon, and argon. Low charge states of magnesium ions are injected into EBIT using a metal-vapor vacuum-arc source. ${ }^{30}$ Neon and argon are injected into EBIT as a gas. Heliumlike ions are formed using the applied quasi-Maxwellian energy sweep. Ions are stored for $\sim 0.5-1 \mathrm{~s}$ and then dumped. Data are collected for most of the time between injections. The resulting photon emission is recorded using two FCSs with thallium hydrogen phthalate $[\mathrm{TAP}(001)]$ crystals for the magnesium and neon data and germanium $[\mathrm{Ge}(111)]$ and ammonium dihydrogen phosphate $[\mathrm{ADP}(101)]$ crystals for argon. Photons are detected using an event-mode data acquisition scheme.

Raw data from a typical quasi-Maxwellian simulation using magnesium are shown as a scatter plot of photon wavelength versus beam energy in Fig. 4. We use here and below the labeling convention of Gabriel ${ }^{31}$ for the heliumlike lines and their $n=2$ DR satellites.

We have measured the intensity of the heliumlike to lithiumlike $n=2$ DR satellite lines $a, b, c, d, j, k, l, q$, and $r$

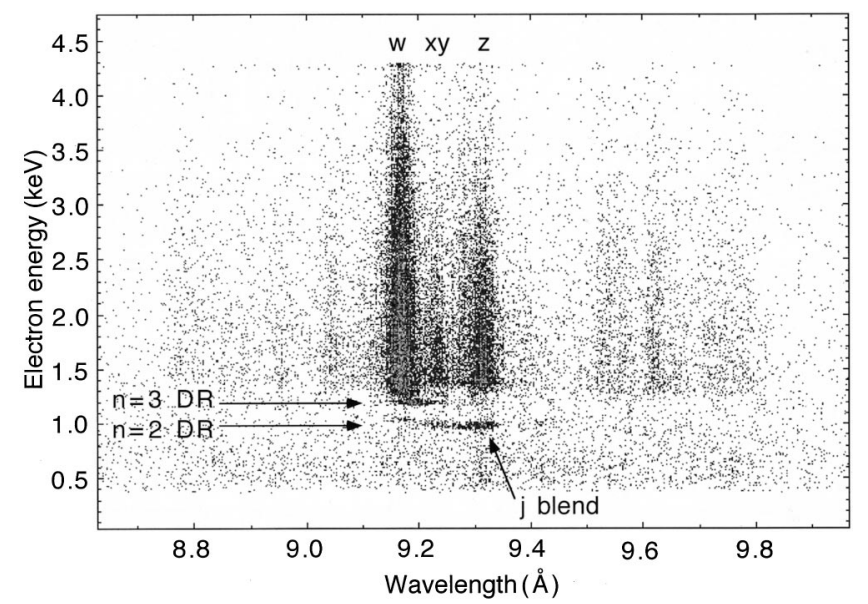

FIG. 4. Scatter plot of photon wavelength vs beam energy for a Maxwellian simulation of $T_{e}=0.7 \mathrm{keV}$. The vertical features above $E \sim 1.35 \mathrm{keV}$ are due to EIE of $\mathrm{Mg}^{10+}$, and are (using the notation of Ref. 31) $w, x$, and $y$ which are blended, and $z$. The features at $E \sim 0.98$ are due to DR into the $n=2$ level of $\mathrm{Mg}^{9+}$. The features at $E \sim 1.2 \mathrm{keV}$ are DR into the $n=3$ level. The tail on $w$ below the EIE threshold energy is due to $n \geqslant 4$ DR.

for $\mathrm{Ne}^{8+}, \mathrm{Mg}^{10+}$, and $\mathrm{Ar}^{16+}$. These lines lie between 13.626 and $13.771 \AA$ for $\mathrm{Ne}^{8+}, 32$ between 9.262 and $9.360 \AA$ for $\mathrm{Mg}^{10+}, 33$ and between 3.982 and $4.004 \AA$ for $\mathrm{Ar}^{16+} .32$ For each ion, these satellite lines are unresolved by our spectrometers. Hereafter, we will refer to this $n=2$ DR feature as $j$, which is its strongest component for each ion. We use the wavelengths from Ref. 34 for $w, x, y$, and $z$ of the ions studied here.

The $n=2$ satellites are formed by electrons with energies of $\sim 0.68 \mathrm{keV}$ for $\mathrm{Ne}^{8+}, \sim 0.98 \mathrm{keV}$ for $\mathrm{Mg}^{10+}$, and $\sim 2.22 \mathrm{keV}$ for $\mathrm{Ar}^{16+}$. The line $w$ is formed by electrons with energies $\geq 0.92 \mathrm{keV}$ for $\mathrm{Ne}^{8+}, \geq 1.35 \mathrm{keV}$ for $\mathrm{Mg}^{10+}$, and $\gtrsim 3.14 \mathrm{keV}$ for $\mathrm{Ar}^{16+}$. We use the event-mode data acquisition technique to select only those $w$ photons produced by EIE. Contributions to $w$ due to DR satellite lines are not included in the measured intensity of $w$.

The measured intensities of $j$ and $w$ are essentially unaffected by contributions due to electron collisions involving lithiumlike and hydrogenic ions. For example, for $\mathrm{Mg}^{9+}$ some of the $n=2$ DR satellites that comprise $j$ can be formed by innershell EIE, but these lines have thresholds $\gtrsim 1.33$ $\mathrm{keV}$. Similarly, innershell electron impact ionization (EII) can produce $z$ which blends with $j$. The threshold for this process is $\gtrsim 1.65 \mathrm{keV} .{ }^{35}$ Using event-mode data acquisition techniques allows us to select only those photons produced at the energy of the $n=2$ DR resonances. A similar procedure is used for the neon and argon data to remove contributions to $j$ and $z$ due to innershell EIE and EII of lithiumlike ions.

RR onto hydrogenic ions can also contribute to $w$ but the contribution is estimated to be small for magnesium ions. Faucher et al $^{36}$ have calculated the effective rate coefficient over a limited temperature range for the production of $w$ due to $\mathrm{RR}$ onto $\mathrm{Mg}^{11+}$. Using their numbers and the total RR rate coefficients of Verner and Ferland, ${ }^{37}$ we estimate that $\sim 9 \%$ of all RR results in the production of a $w$ photon. We estimate the relative $\mathrm{Mg}^{11+} / \mathrm{Mg}^{10+}$ abundance from measurements of the $\mathrm{Mg}^{11+} \operatorname{Ly} \alpha\left(1 s^{2} S_{1 / 2}-2 p^{2} P_{3 / 2,1 / 2}\right)$ and 
TABLE II. Summary of percentage uncertainties for $j / w$ line ratio measurements. All uncertainties are quoted at a confidence level considered to be equivalent to a $1 \sigma$ statistical confidence level.

\begin{tabular}{lcccccccccccc}
\hline \hline & \multicolumn{11}{c}{$T_{e}(\mathrm{keV})$} \\
\cline { 2 - 13 } \multicolumn{1}{c}{ Source } & 0.2 & 0.4 & 0.5 & 0.6 & 0.7 & 1.0 & 1.4 & 1.5 & 1.8 & 2.0 & 2.2 & 2.4 \\
\hline Element & $\mathrm{Ne}$ & $\mathrm{Ne}$ & $\mathrm{Mg}$ & $\mathrm{Ne}$ & $\mathrm{Mg}$ & $\mathrm{Mg}$ & $\mathrm{Ar}$ & $\mathrm{Mg}$ & $\mathrm{Ar}$ & $\mathrm{Mg}$ & $\mathrm{Ar}$ & $\mathrm{Ar}$ \\
Line ratio & 8 & 8 & 4 & 7 & 6 & 11 & 7 & 7 & 9 & 23 & 9 & 9 \\
Radiative recombination & 1 & 1 & 1 & 1 & 1 & 1 & 1 & 1 & 1 & 1 & 1 & 1 \\
Overlap integral & 11 & 11 & 6 & 11 & 6 & 7 & 8 & 7 & 8 & 8 & 8 & 8 \\
Transmittance & 1 & 1 & 1 & 1 & 1 & 1 & 1 & 1 & 1 & 1 & 1 & 1 \\
Detection efficiency & 1 & 1 & 1 & 1 & 1 & 1 & 1 & 1 & 1 & 1 & 1 & 1 \\
$G_{w}^{\prime} / G_{j}^{\prime}$ & 2 & 4 & 3 & 7 & 4 & 4 & 3 & 5 & 4 & 6 & 4 & 4 \\
Quadrature sum & 14 & 14 & 8 & 15 & 10 & 14 & 11 & 11 & 13 & 25 & 13 & 13 \\
\hline \hline
\end{tabular}

$\mathrm{Mg}^{10+} K \beta\left(1 s^{2}{ }^{1} S_{0}-1 s 3 p{ }^{1} P_{1}\right)$ intensities carried out simultaneously with the quasi-Maxwellian simulations. The inferred $\mathrm{Mg}^{11+} / \mathrm{Mg}^{10+}$ abundances are over five times smaller than that predicted for a plasma in ionization equilibrium. ${ }^{38,39}$ This difference is most likely due to the effects of charge transfer (CT) on the ionization balance in EBIT. Scaling the RR rate coefficients of Verner and Ferland ${ }^{37}$ by 0.09 , using the theoretical rate coefficient for production of $w$ due to $\mathrm{EIE},{ }^{36}$ and reducing by a factor of 5 the $\mathrm{Mg}^{11+} / \mathrm{Mg}^{10+}$ abundance from the ionization balance calculations of Arnaud and Rothenflug, ${ }^{39}$ we estimate that the RR contribution to $w$ is $<1 \%$ over the simulated temperature range.

For both the neon and argon data, we have estimated the hydrogenic-to-heliumlike relative abundances by measuring the intensities of $\operatorname{Ly} \alpha$ and $w$ simultaneously with the quasiMaxwellian simulations. The inferred relative abundances are over 10 times smaller than predicted for a plasma in ionization equilibrium. ${ }^{38,39}$ Again, this is most likely due to CT effects in EBIT. Scaling the RR rate coefficients of Verner and Ferland ${ }^{37}$ by 0.09 , using the theoretical rate coefficient for production of $w$ due to EIE, ${ }^{40}$ and reducing by a factor of 10 the hydrogenic-to-heliumlike relative abundance from the ionization balance calculations of Arnaud and Rothenflug, ${ }^{39}$ we estimate that for both data sets the RR contribution to $w$ is $<1 \%$ over the temperature ranges simulated.

CT of hydrogenic ions with background gas in EBIT and $\mathrm{RR}$ with beam electrons is responsible for producing $z$ at energies below the EIE threshold. We subtract the CT+RR background using the measured background level for energies below and above the $n=2$ DR resonance energy. CT of hydrogenic ions also contributes to $w$. We subtract this contribution to $w$ using the measured background level below the EIE threshold and away from all DR resonances. During a Maxwellian simulation, the time spent at each beam energy is not constant. We account for this variation when we determine the backgrounds to be subtracted for both $j$ and $w$.

Statistical uncertainties in the measured $j / w$ line ratio are listed in Table II. Uncertainties are quoted at a $1 \sigma$ confidence level. The lines $j$ and $w$ are formed at different energies. Taking into account the energies at which these lines form, there may be an $\sim 11 \%$ uncertainty for the neon data, an $\sim 8 \%$ uncertainty for the magnesium data, and an $\sim 8 \%$ for the argon data which is introduced when the overlap integrals are canceled out in Eq. (21).

\section{Effects of $E_{\min }$ and $E_{\max }$}

The lower energy limit of the sweep, $E_{\min }$, results in a RR rate smaller than one would expect for a true Maxwellian plasma. But because we subtract the contributions to $j$ and $w$ due to RR of hydrogenic ions, this error has an insignificant effect on the measured line intensities. $E_{\min }$ has no effect on line emission due to DR or EIE as all the relevant DR resonances and EIE thresholds lie above the values of $E_{\min }$ used. The reduced RR rate might also affect the charge balance of the trap were it not for CT which dominates the total recombination rate in EBIT.

The upper energy limit, $E_{\max }$, results in EII and EIE rates which are smaller than the rates for a true Maxwellian plasma. This difference has no effect on the measured intensity of $j$. As discussed in Sec. IV B, event-mode data acquisition techniques allow us to remove line emission due to EII and inner shell EIE of lithiumlike ions from the measured intensity of $j$.

We are unaware of any published theoretical cross sections for EIE production of $w$ for $\mathrm{Ne}^{8+}, \mathrm{Mg}^{10+}$, or $\mathrm{Ar}^{16+}$. So we use the calculations of Ref. 41 for $\mathrm{Ti}^{20+}$ to estimate the effects of $E_{\max }$ on the quasi-Maxwellian EIE rate coefficients for $w$. To integrate the cross sections of Ref. 41 we have fitted them using Eq. (26) of Ref. 42. Energy levels scale as $q^{2}$, so we have scaled the simulated temperatures and values of $E_{\max }$ by $25 / 4,4$, and 25/16 to use the $\mathrm{Ti}^{20+}$ results for $\mathrm{Ne}^{8+}, \mathrm{Mg}^{10+}$, and $\mathrm{Ar}^{16+}$, respectively. We find that the quasi-Maxwellian rates for $w$ are $\sim 3 \%-7 \%$ smaller than the true Maxwellian rates for every ion and temperature except for $\mathrm{Ne}^{8+}$ at $0.2 \mathrm{keV}$ where the difference is $\sim 18 \%$. We have increased the measured intensities of $w$ to account for the estimated contributions lost due to $E_{\max }$. The effect of $E_{\max }$ on the simulated Maxwellian can be reduced by using a power supply capable of providing more current. Making this change would allow slew rates of $d E / d t \geq 30$ $\mathrm{V} \mathrm{cm}^{-1}$, thereby increasing the $E_{\max }$ achievable, which in turn would extend the high energy portion of the Maxwellian distribution which is simulated.

We have calculated the quasi-Maxwellian EII rates using the recommended EII cross sections of Ref. 43. The resulting 
rates for ionization of neutral through lithiumlike neon, magnesium, and argon are $\sim 2 \%$ smaller than the true Maxwellian rates for the temperatures simulated. The smaller rates reduce insignificantly the time required to ionize the plasma up to heliumlike. The EII rates for the heliumlike and hydrogenic ions are $\sim 3 \%-35 \%$ smaller than the true Maxwellian rates for the temperatures simulated, except for neon at 0.2 $\mathrm{keV}$ where the quasi-Maxwellian EII rates are essentially zero. The smaller EII rates for the heliumlike and hydrogenic ions reduce somewhat the final charge balance achieved. The magnitude of this effect, though, is small compared to that of $\mathrm{CT}$ on the ionization state of the plasma.

\section{Timescales}

The heliumlike ions in the trap were generally produced using the quasi-Maxwellian simulation, though in a few cases they were produced using a monoenergetic beam. Electron densities of $(1-5) \times 10^{12} \mathrm{~cm}^{-3}$ were used for the simulations. The ions in the trap oscillate in and out of the electron beam and are estimated to spend only about a third of the time in the beam. ${ }^{44}$ The effective electron density seen by the ions is reduced accordingly.

With the recommended EII rate coefficients of Ref. 45, which are based on the cross sections of Ref. 43, we can estimate the time required to ionize up to the heliumlike charge state. Using the appropriate electron densities for the temperatures simulated and the rates of Ref. 45 , we estimate that the plasma ionizes to heliumlike in 6-75 ms.

Typical ionization times before beginning data acquisition were 9-16 ms. This time is, in general, shorter than the time required to reach the heliumlike charge states. However, as discussed, in Sec. IV B, line emission from charge states other than heliumlike has an insignificant effect on the present results.

The ionization time of the heliumlike ions is estimated to vary between 0.06 and $4.28 \mathrm{~s}$. This time is significantly longer than the $10 \mathrm{~ms}$ period for the down-up-down sweep of the quasi-Maxwellian. Thus, changes in the heliumlike charge balance during the course of a sweep are estimated to have an insignificant effect on the observed line emission.

\section{E. Spectrometer efficiency}

Relative transmittances and detector efficiencies for $j$ and $w$ are calculated using the photoabsorption cross section of Ref. 46. The uncertainties in the relative transmittances and detector efficiencies are insignificant. The values of $R_{\sigma}$ and $R_{\pi}$ have been determined using the limiting theoretical values for the total integrated reflectivity $R$ and for $R_{\pi} / R_{\sigma}$ for both a perfect crystal with non-negligible absorption and for a mosaic crystal. ${ }^{46}$ Using $R=\left(R_{\sigma}+R_{\pi}\right) / 2$, we have calculated $R_{\pi}$ and $R_{\sigma}$ for $j$ and $w$ for these two limiting cases.

We have estimated $G_{w}^{\prime}$ [see Eq. (20)] using the cross sections and polarization factors for heliumlike $\mathrm{Ti}^{20+}$ from Ref. 41. This approximation is justified because the polarization of $w$ vs $E$ for $Z \lesssim 22$ is nearly independent of $Z .{ }^{41}$ The atomic code used in Ref. 41 is in good agreement with measured cross sections ${ }^{47,48}$ and polarization factors ${ }^{23,49-51}$ for $w$ of several heliumlike ions. For use in calculating Eq. (19), we have fit the cross sections and polarization factors of Ref. 41 using Eq. (26) of Ref. 42 . We have also reduced the $\mathrm{Ti}^{20+}$ temperature scale by $4 / 25,1 / 4$, and $16 / 25$ to use the $\mathrm{Ti}^{20+}$ $G_{w}^{\prime}$ results for $\mathrm{Ne}^{8+}, \mathrm{Mg}^{10+}$, and $\mathrm{Ar}^{16+}$, respectively.

Before calculating $G_{w}^{\prime}$ for $\mathrm{Ti}^{20+}$, it is important that the value used for $E_{\perp}$ is appropriately scaled to the ion for which the calculations will ultimately be used. This procedure is done by scaling $E_{\perp}$ by $25 / 4,4$, and $25 / 16$ for $\mathrm{Ne}^{8+}, \mathrm{Mg}^{10+}$, and $\mathrm{Ar}^{16+}$, respectively, before calculating $G_{w}^{\prime}$.

The value of $G_{j}^{\prime}$ is calculated using Eq. (20). Theoretical polarizations for $a, b, c, d, j, k, l, q$, and $r$ are taken from Ref. 52. Resonance strengths for these lines are taken from Refs. 33, 39, and 53 for magnesium and from Refs. 32, 53, and 54 for neon and argon. Variations between the different theoretical resonance strengths have an insignificant effect on $G_{j}^{\prime}$.

We calculate $G_{w}^{\prime} / G_{j}^{\prime}$ using the two limiting crystal cases and $E_{\perp}=0,200$, and $400 \mathrm{eV}$. We use the resulting mean value. This introduces an error of $\$ 6 \%$ for the magnesium data, $\$ 7 \%$ for the neon data, and $\$ 4 \%$ for the argon data.

\section{THEORY}

The relevant DR resonance strengths have been calculated by a number of different groups. For magnesium we use the calculations of Refs. 32, 33, and 53 and for neon and argon of Refs. 32, 53, and 54. For neon, we have shifted the wavelengths of Ref. 54 by $-0.02 \AA$ so the $n=2$ wavelengths are in better agreement with those of Refs. 32 and 53. For the strongest lines in the $j$ feature, the calculations differ among themselves by $\sim 20 \%$ for neon, $\sim 15 \%$ for magnesium, and $\sim 14 \%$ for argon. We calculate the total resonance strength of the $j$ blend by taking the mean values from the different theoretical calculations of the relevant resonance strengths. We estimate there is an $\sim 10 \%, \sim 8.5 \%$, and $\sim 7 \%$ uncertainty in the resulting $C_{j}$ for neon, magnesium, and argon, respectively.

The rate coefficient for $\mathrm{Mg}^{10+} w\left(C_{w}\right)$ has been calculated by Faucher et al. ${ }^{36}$ by Zhang and Sampson, ${ }^{40}$ and by us. The calculations of Faucher et al. include estimates for cascades from $n \geqslant 3$ but do not include the effects of resonances. The calculations of Zhang and Sampson include the effects of resonances but do not include cascades. We have carried out new calculations using the Hebrew University/ Lawrence Livermore Atomic Code (HULLAC) which is described in Ref. 55 and references therein. Our HULLAC calculations (Table III) include cascade contributions from $n$ $\leqslant 6$ levels but do not include resonance effects. The calculations of Faucher et al. were carried out only for $T_{e} \lesssim 0.78$ $\mathrm{keV}$. Between 0.50 and $0.78 \mathrm{keV}$, their calculations are $\sim 10 \%$ smaller than those of Zhang and Sampson. The HULLAC results are $\sim 7 \%$ larger than those of Zhang and Sampson. This difference is probably the result of including cascades which increased the HULLAC results by $\sim 6 \%$. We use $C_{w}$ from Zhang and Sampson and estimate that there is a $+7 \%$ to $-10 \%$ uncertainty. To calculate $C_{w}$ at temperatures not given, we have fit the data of Zhang and Sampson using Eq. (27) of Ref. 42. For neon and argon we use $C_{w}$ of Zhang and Sampson and again assume a $+7 \%$ to $-10 \%$ uncertainty. 
TABLE III. HULLAC EIE rate coefficients for producing $\mathrm{Mg}^{10+} w$. Cascade contributions from $n \leqslant 6$ levels are included.

\begin{tabular}{cc}
\hline \hline $\begin{array}{c}T_{e} \\
(\mathrm{keV})\end{array}$ & $\begin{array}{c}C_{w} \\
\left(\mathrm{~cm}^{3} \mathrm{~s}^{-1}\right)\end{array}$ \\
\hline 89.3 & $2.67 e-17$ \\
100 & $1.29 e-16$ \\
134 & $3.60 e-15$ \\
200 & $8.87 e-14$ \\
300 & $7.61 e-13$ \\
447 & $3.09 e-12$ \\
500 & $4.18 e-12$ \\
700 & $8.52 e-12$ \\
1000 & $1.47 e-11$ \\
1500 & $2.24 e-11$ \\
2000 & $2.76 e-11$ \\
2500 & $3.13 e-11$ \\
\hline \hline
\end{tabular}

\section{RESULTS AND DISCUSSION}

We have measured the quasi-Maxwellian $j / w$ line ratio for neon, magnesium, and argon (Fig. 5). All data are shown with their estimated $1 \sigma$ error bars. The ratios are plotted assuming that the simulated $T_{e}$ matches the true Maxwellian $T_{e}$. Uncertainties are listed in Table II. Uncertainties are treated as random sign errors and added in quadrature. Table IV lists the temperatures simulated, the ion used, the electron energy of the $n=2$ DR resonances, the threshold energy of $w$, the values of $E_{\min }$ and $E_{\max }$ used, and the length of time for which data were collected.

In Fig. 5, we have plotted the best guess theoretical value of $j / w$ vs $T_{e}$ and the estimated range of theoretical ratios. Although agreement between theory and experiment is excellent, in general our measured $j / w$ ratio falls on or below the best-guess theoretical $j / w$ ratio. Experimentally, this trend might indicate that we are undersampling the electron energy distribution at energies around the $n=2$ DR resonances and/or oversampling the distribution at energies above the threshold for excitation of $w$. Theoretically, this trend might indicate the best-guess value of $C_{j}$ is too high and/or that of $C_{w}$ too low. In fact, for all three elements, our measured ratios are in best agreement with the theoretical $j / w$ ratio calculated using the results of $\mathrm{Chen}^{32}$ for $C_{j}$, which yield the smallest values of $C_{j}$, and the HULLAC results for $C_{w}$, which yield the largest values of $C_{w}$ (i.e., essentially the lower dotted curves in Fig. 5). This latter suggestion is partly supported by recent DR measurements in heliumlike $\mathrm{Fe}^{24+}{ }^{8}$ which found best agreement with the calculations of Chen compared to the theory of Refs. 53, 54, and 56 (which uses the same theoretical techniques as Refs. 33 and 36).

We use the measured values of $j / w$ and the range of theoretical $j / w$ values versus $T_{e}$ to infer $T_{e}$ of the observed quasi-Maxwellian plasmas. Figure 6 shows the inferred $T_{e}$ as a function of the simulated $T_{e}$. The fidelity of the quasiMaxwellian simulation is shown in Fig. 7 which plots the ratio of the difference between the inferred and simulated $T_{e}$ to the simulated $T_{e}$. Figure 7 shows that the inferred values of $T_{e}$ agree on average to within better than $10 \%$ with the simulated values. This agreement demonstrates the success
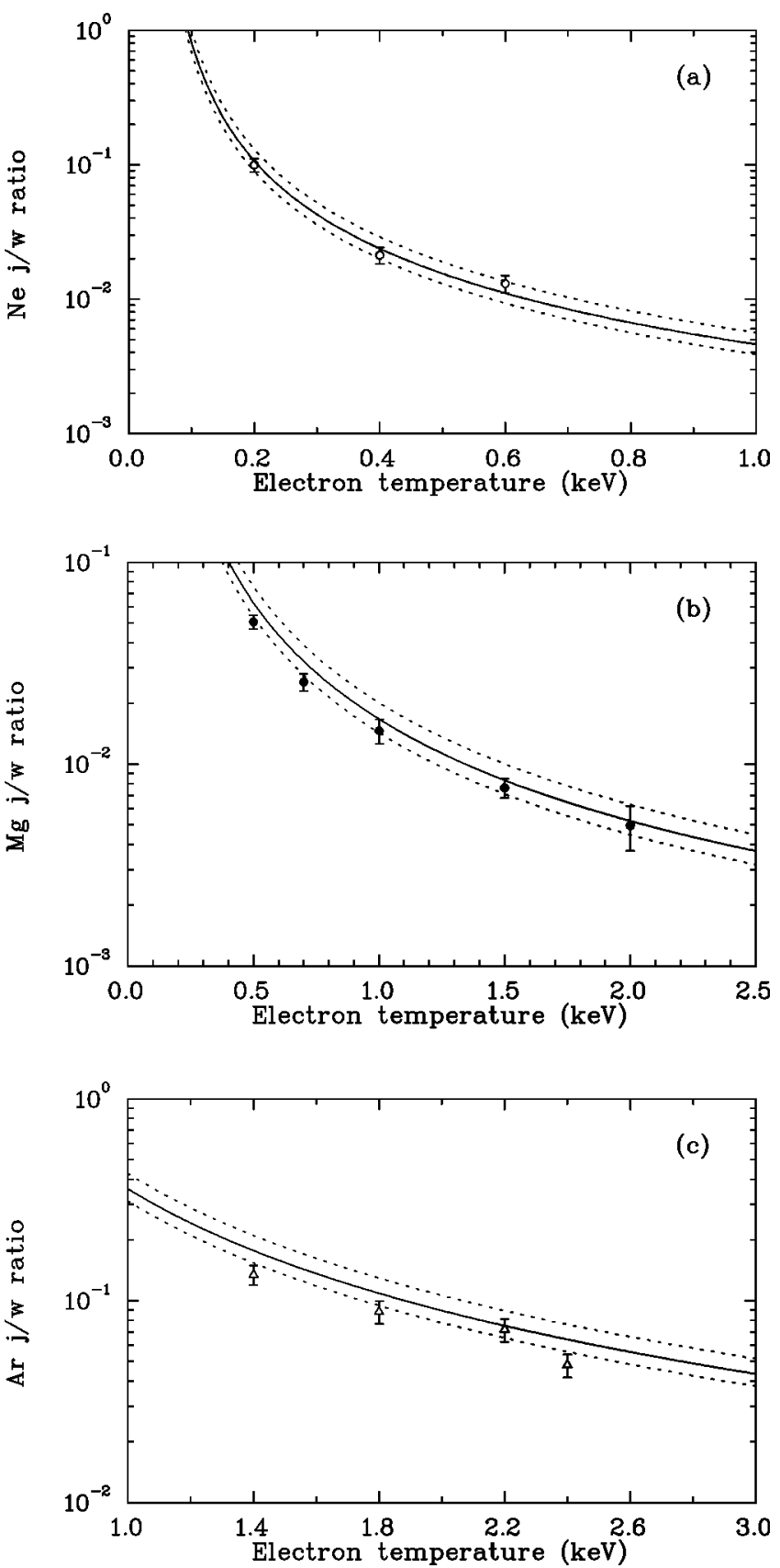

FIG. 5. Measured and theoretical $j / w$ line ratio vs $T_{e}$ for (a) neon, (b) magnesium, and (c) argon. Experimental results are shown with their estimated $1 \sigma$ confidence limits. The solid curve is the best-guess theoretical line ratio. The dotted curves show the estimated range of the theoretical ratios (see the text).

of our new technique for simulating a Maxwell-Boltzmann electron distribution.

\section{ACKNOWLEDGMENTS}

The authors wish to thank J. Crespo López-Urrutia and R. E. Marrs for stimulating conversation and P. A. D'Antonio, E. W. Magee, and D. H. Nelson for their expert technical support. This program was supported by NASA High Energy Astrophysics X-Ray Astronomy Research and Analysis Grant No. NAG5-5123 (Columbia University) and work Order No. W-19127 (Lawrence Livermore National Laboratory). Work performed at Lawrence Livermore Na- 
TABLE IV. Summary of run conditions. Listed are the Maxwellian temperatures simulated (column 1), the ions used (column 2), the electron energy of the $n=2$ DR resonances ( $E_{2}$, column 3 ), the threshold excitation energy for $w\left(E_{w}\right.$, column 4$), E_{\min }$ and $E_{\max }$ of the beam energy sweep (columns 5 and 6 , respectively), and the number of hours for which data were collected (column 7)

\begin{tabular}{ccccccc}
\hline \hline $\begin{array}{c}T_{e} \\
(\mathrm{keV})\end{array}$ & Ion & $\begin{array}{c}E_{2} \\
(\mathrm{keV})\end{array}$ & $\begin{array}{c}E_{w} \\
(\mathrm{keV})\end{array}$ & $\begin{array}{c}E_{\min } \\
(\mathrm{keV})\end{array}$ & $\begin{array}{c}E_{\max } \\
(\mathrm{keV})\end{array}$ & $\begin{array}{c}\text { Time } \\
(\mathrm{h})\end{array}$ \\
\hline 0.2 & $\mathrm{Ne}^{8+}$ & 0.68 & 0.92 & 0.20 & 1.20 & 24 \\
0.4 & $\mathrm{Ne}^{8+}$ & 0.68 & 0.92 & 0.20 & 2.90 & 15 \\
0.5 & $\mathrm{Mg}^{10+}$ & 0.98 & 1.35 & 0.25 & 3.00 & 12 \\
0.6 & $\mathrm{Ne}^{8+}$ & 0.68 & 0.92 & 0.20 & 3.60 & 13 \\
0.7 & $\mathrm{Mg}^{10+}$ & 0.98 & 1.35 & 0.20 & 4.20 & 16 \\
1.0 & $\mathrm{Mg}^{10+}$ & 0.98 & 1.35 & 0.25 & 6.00 & 15 \\
1.4 & $\mathrm{Ar}^{16+}$ & 2.22 & 3.14 & 0.60 & 7.98 & 33 \\
1.5 & $\mathrm{Mg}^{10+}$ & 0.98 & 1.35 & 0.20 & 8.40 & 23 \\
1.8 & $\mathrm{Ar}^{16+}$ & 2.22 & 3.14 & 0.50 & 9.80 & 19 \\
2.0 & $\mathrm{Mg}^{10+}$ & 0.98 & 1.35 & 0.45 & 10.60 & 2 \\
2.2 & $\mathrm{Ar}^{16+}$ & 2.22 & 3.14 & 0.60 & 11.44 & 16 \\
2.4 & $\mathrm{Ar}^{16+}$ & 2.22 & 3.14 & 0.60 & 12.24 & 18 \\
\hline \hline
\end{tabular}

tional Laboratory was performed under the auspices of the U.S. Department of Energy (Contract No. W-7405-ENG-48).

\section{APPENDIX: DERIVATION OF EQ. (3)}

We wish to solve

$$
\tau(E)=\tau_{0} \int_{0}^{E} P\left(E^{\prime}, T_{e}\right) d E^{\prime},
$$

where $P\left(E^{\prime}, T_{e}\right)$ is given by Eq. (1). Setting $\alpha$ $=2 \pi^{-1 / 2}\left(k_{B} T_{e}\right)^{-3 / 2}, \quad \beta=1 / k_{B} T_{e}, x^{2}=\beta E^{\prime}, \quad y=x^{2} / \beta$, and dropping the primes gives

$$
\tau(E)=\frac{2 \alpha \tau_{0}}{\beta^{3 / 2}} \int_{0}^{y} x^{2} e^{-x^{2}} d x .
$$

Equation (A2) can be solved by twice integrating by parts. The first integration yields



FIG. 6. Spectroscopically inferred $T_{e}$ (using $j / w$ ) plotted as a function of the quasi-Maxwellian simulated $T_{e}$. Data are shown for neon (open circles), magnesium (closed circles), and argon (open triangles). The error bars represent the combined effects of the $1 \sigma$ experimental uncertainties and the range of theoretical $j / w$ ratios. The straight line shows $\left(T_{e}\right)_{\text {inferred }}$ $=\left(T_{e}\right)_{\text {simulated }}$.

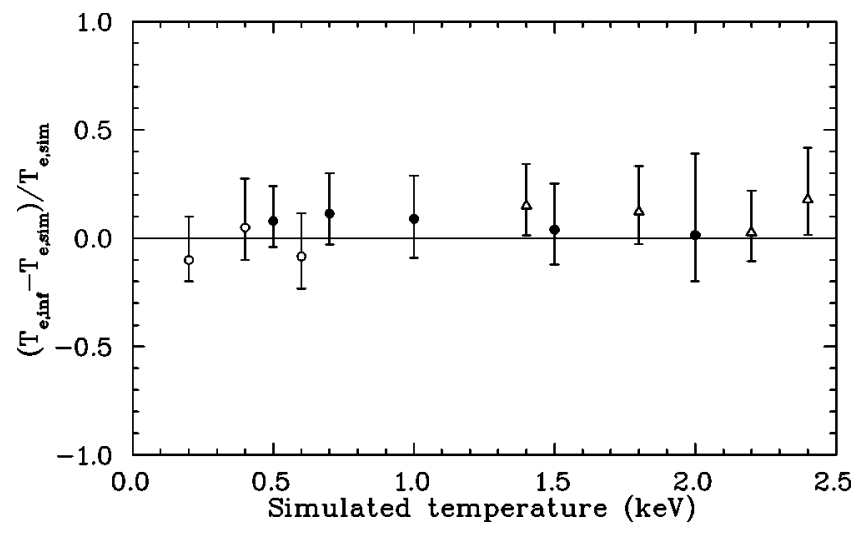

FIG. 7. Fidelity of the quasi-Maxwellian simulation. Ratio of the difference between the simulated and inferred $T_{e}$ to the simulated value.

$$
\int x^{2} e^{-x^{2}} d x=\frac{\sqrt{\pi}}{2} x^{2} \operatorname{erf}(x)-\sqrt{\pi} \int x \operatorname{erf}(x) d x,
$$

where $\operatorname{erf}(x)$ is the error function. Then integrating by parts the integral on the right-hand side of Eq. (A3) yields

$$
\int x \operatorname{erf}(x) d x=\frac{x^{2} \operatorname{erf}(x)}{2}+\frac{x e^{-x^{2}}}{2 \sqrt{\pi}}-\frac{1}{4} \operatorname{erf}(x),
$$

where we have made use of ${ }^{57}$

$$
\int \operatorname{erf}(x) d x=x \operatorname{erf}(x)+\frac{e^{-x^{2}}}{\sqrt{\pi}} .
$$

Substituting Eqs. (A3) and (A4) into Eq. (A2) we find

$$
\tau(E)=\tau_{0}\left[\operatorname{erf}(x)-\frac{2 x e^{-x^{2}}}{\sqrt{\pi}}\right] .
$$

${ }^{1}$ H. R. Griem, J. Quant. Spectrosc. Radiat. Transf. 40, 403 (1988).

${ }^{2}$ M. A. Levine, R. E. Marrs, J. R. Henderson, D. A. Knapp, and M. B. Schneider, Phys. Scr., T 22, 157 (1988).

${ }^{3}$ M. A. Levine et al., Nucl. Instrum. Methods Phys. Res. B 43, 431 (1989).

${ }^{4}$ J. Dubau and S. Volonté, Rep. Prog. Phys. 43, 199 (1980).

${ }^{5}$ G. R. Brewer, in Focusing of Charged Particles, edited by by A. Septier (Academic, New York, 1967), p. 23.

${ }^{6}$ D. A. Knapp, R. E. Marrs, S. R. Elliot, E. W. Magee, and R. Zasadzinski, Nucl. Instrum. Methods Phys. Res. A 334, 305 (1993).

${ }^{7}$ S. B. Utter, P. Beiersdorfer, J. Crespo López-Urrutia, K. Widmann, and E. J. Clothiaux, Nucl. Instrum. Methods Phys. Res. A 428, 276 (1999).

${ }^{8}$ P. Beiersdorfer, T. W. Phillips, K. L. Wong, R. E. Marrs, and D. A. Vogel, Phys. Rev. A 46, 3812 (1992).

${ }^{9}$ M. F. Gu, S. M. Kahn, D. W. Savin, P. Beiersdorfer, G. V. Brown, D. A. Liedahl, K. J. Reed, C. P. Bhalla, and S. R. Grabbe, Astrophys. J. 518, 1002 (1999).

${ }^{10}$ D. Vogel, Lawrence Livermore National Laboratory Internal Report No. UCRL-ID-104990 (1990).

${ }^{11}$ P. Beiersdorfer and B. J. Wargelin, Rev. Sci. Instrum. 65, 13 (1994).

${ }_{12}$ P. Beiersdorfer, J. R. Crespo López-Urrutia, E. Förster, J. Mahiri, and K. Widmann, Rev. Sci. Instrum. 68, 1077 (1997).

${ }^{13}$ G. V. Brown, P. Beiersdorfer, and K. Widmann, Rev. Sci. Instrum. 70, 280 (1999).

${ }^{14}$ C. J. Borkowski and M. K. Kopp, Rev. Sci. Instrum. 39, 1515 (1968).

${ }^{15}$ I. C. Percival and M. J. Seaton, Philos. Trans. R. Soc. London, Ser. A 251, 113 (1958).

${ }^{16}$ U. Fano and J. H. Macek, Rev. Mod. Phys. 45, 553 (1973).

${ }^{17}$ M. K. Inal and J. Dubau, J. Phys. B 20, 4221 (1987).

${ }^{18}$ D. W. Savin, P. Beiersdorfer, J. Crespo López-Urrutia, V. Decaux, E. M. Gullikson, S. M. Kahn, D. A. Liedahl, K. J. Reed, and K. Widmann, Astrophys. J. 470, L73 (1996). 
${ }^{19}$ D. W. Savin, M. F. Gu, and P. Beiersdorfer, in Proceedings of the Japan-US Workshop on Plasma Polarization Spectroscopy and The International Seminar on Plasma Polarization Spectroscopy, Kyoto, Japan, 1998, edited by T. Fujimoto and P. Beiersdorfer, National Institute for Fusion Science Research Report No. NIFS-PROC-37, p. 90.

${ }^{20}$ M. F. Gu, D. W. Savin, and P. Beiersdorfer, J. Phys. B 32, 5371 (1999).

${ }^{21}$ J. D. Jackson, Classical Electrodynamics (Wiley, New York, 1975), p. 588.

${ }^{22}$ S. Pastuszka et al., Nucl. Instrum. Methods Phys. Res. A 369, 11 (1996).

${ }^{23}$ P. Beiersdorfer et al., Phys. Rev. A 53, 3974 (1996).

${ }^{24}$ D. A. Knapp, R. E. Marrs, M. B. Schneider, M. H. Chen, and M. A. Levine, Phys. Rev. A 47, 2039 (1993).

${ }^{25}$ J. H. Scofield, Phys. Rev. A 40, 3054 (1989).

${ }^{26}$ J. H. Scofield, Phys. Rev. A 44, 139 (1991).

${ }^{27}$ E. B. Saloman, J. H. Hubbell, and J. H. Scofield, At. Data Nucl. Data Tables 38, 1 (1988).

${ }^{28}$ R. E. Marrs, P. Beiersdorfer, S. R. Elliott, D. A. Knapp, and Th. Stoehlker, Phys. Scr., T 59, 183 (1995).

${ }^{29}$ B. M. Penetrante, J. N. Bardsley, D. DeWitt, M. Clark, and D. Schneider, Phys. Rev. A 43, 4861 (1991).

${ }^{30}$ I. G. Brown, J. E. Galvin, R. A. MacGill, and R. T. Wright, Appl. Phys. Lett. 49, 1019 (1986).

${ }^{31}$ A. H. Gabriel, Mon. Not. R. Astron. Soc. 160, 99 (1972).

${ }^{32}$ M. H. Chen, At. Data Nucl. Data Tables 34, 301 (1986).

${ }^{33}$ L. Steenman-Clark, F. Bely-Dubau, and P. Faucher, Mon. Not. R. Astron. Soc. 191, 951 (1980).

${ }^{34}$ D. R. Plante, W. R. Johnson, and J. Sapirstein, Phys. Rev. A 49, 3519 (1994).

${ }^{35}$ W. Lotz, J. Opt. Soc. Am. 58, 915 (1968).

${ }^{36}$ P. Faucher, M. Loulergue, L. Steenman-Clark, and S. Volonté, Astron. Astrophys. 118, 147 (1983).

${ }^{37}$ D. A. Verner and G. J. Ferland, Astrophys. J., Suppl. Ser. 103, 476 (1996).

${ }^{38}$ R. Mewe, E. H. B. M. Gronenschild, and G. H. J. van den Oord, Astron. Astrophys., Suppl. Ser. 62, 197 (1985).
${ }^{39}$ M. Arnaud and R. Rothenflug, Astron. Astrophys., Suppl. Ser. 60, 425 (1985).

${ }^{40}$ H. Zhang and D. H. Sampson, Astrophys. J., Suppl. Ser. 63, 487 (1987).

${ }^{41}$ K. J. Reed and M. H. Chen, Phys. Rev. A 48, 3644 (1993).

${ }^{42}$ R. Mewe and E. H. B. M. Gronenschild, Astron. Astrophys., Suppl. Ser. 45, 11 (1981).

${ }^{43}$ M. A. Lennon, K. L. Bell, H. B. Gilbody, J. G. Hughes, A. E. Kingston, M. J. Murray, and F. J. Smith, J. Phys. Chem. Ref. Data 3, 1285 (1988).

${ }^{44}$ V. Decaux, P. Beiersdorfer, S. M. Kahn, and V. L. Jacobs, Astrophys. J. 482, 1076 (1997).

${ }^{45}$ G. S. Voronov, At. Data Nucl. Data Tables 65, 1 (1997).

${ }^{46}$ B. L. Henke, E. M. Gullikson, and J. C. Davis, At. Data Nucl. Data Tables 54, 181 (1993); these data are available at http://www-cxro.lbl.gov/ optical_constants.

${ }^{47}$ S. Chantrenne, P. Beiersdorfer, R. Cauble, and M. B. Schneider, Phys. Rev. Lett. 69, 265 (1992).

${ }^{48}$ K. L. Wong, P. Beiersdorfer, K. J. Reed, and D. A. Vogel, Phys. Rev. A 51, 1214 (1995).

${ }^{49}$ J. R. Henderson et al., Phys. Rev. Lett. 65, 705 (1990).

${ }^{50}$ P. Beiersdorfer, in Ref. 19, p. 67.

${ }^{51}$ P. Beiersdorfer, G. Brown, S. Utter, P. Neill, K. J. Reed, A. J. Smith, and R. S. Thoe, Phys. Rev. A 60, 4356 (1999).

${ }^{52}$ M. K. Inal and J. Dubau, J. Phys. B 22, 3329 (1989).

${ }^{53}$ L. A. Vainshtein and U. I. Safronova, At. Data Nucl. Data Tables 21, 49 (1978).

${ }^{54}$ J. Nilsen, At. Data Nucl. Data Tables 38, 339 (1988).

${ }^{55}$ D. A. Liedahl, A. L. Osterheld, and W. H. Goldstein, Astrophys. J. 438, L115 (1995).

${ }^{56}$ F. Bely-Dubau, J. Dubau, P. Faucher, and A. H. Gabriel, Mon. Not. R. Astron. Soc. 198, 239 (1982).

${ }^{57}$ I. S. Gradshteyn and I. M. Ryzhik, Table of Integrals, Series, and Products (Academic, New York, 1965), p. 663. 